\title{
Os desafios dos serviços psicológicos mediados pelas TIC no contexto da Pandemia do Coronavírus 2019-2020
}

\section{Sérgio Rodrigues de Santana}

Psicólogo. Doutorando em Ciência da Informação pela Universidade Federal da Paraíba.

sergiokafe@hotmail.com

Carla Daniella Teixeira Girard

Bibliotecária na Universidade Federal Rural da Amazônia. Doutoranda em Educação pela Universidade Luterana do Brasil.

carlinhagirard@yahoo.com.br

Levi Cadmiel Amaral da Costa

Administrador. Mestrando em Ciência da Informação pela Universidade Federal da Paraíba.

levy.cadmiel@gmail.com

Cristiane Marina Teixeira Girard

Bibliotecária na Universidade Federal de

Rondônia. Mestra em Letras pela

Universidade Federal de Rondônia.

marinateixeiragirard@gmail.com

Daniel Jackson Estevam da Costa

Doutor em Química pela Universidade Federal da Paraíba.

danieljacksonpb@gmail.com

\section{Resumo}

No contexto da Pandemia do Coronavírus 2019-20 os serviços psicológicos mediados pelas Tecnologia da Informação e Comunicação se tornaram essenciais para o equilíbrio da saúde mental. Estes serviços são atividades clínicas realizadas por psicológicos cadastros nos Conselhos Federal e Regionais de Psicologia através do e-Psi. Objetiva refletir de forma crítica sobre o papel da tecnologia quanto aos atendimentos psicológicos mediados pelas Tecnologias da Informação e Comunicação no contexto da Pandemia do Coronavírus 2019-2020. Teve abordagem qualitativa, o método descritivocompreensivo-interpretativo, inclinação interdisciplinar de autores e a coleta de dados de fontes primárias por meio de documentos legislativos, normativos, científicos e informativos publicados no site do Conselho Federal de Psicologia. O aporte teórico da Ciência da Informação se apoiou nas teorias da Revolução da Tecnologia da Informação e Filosofia da Tecnologia. Ao considerar a relação tecnologia, Psicologia, comportamento humano e serviços clínicos a Psicologia utiliza teorias clássicas na interpretação de novos construtos comportamentais e sociais originados na Sociedade da Informação e do Conhecimento. Entre 1995 a 2012 na perspectiva histórica-epistémico houve avanços significativos acerca dos estudos teóricos, técnicos e epistêmicos sobre os atendimentos psicológicos mediados pelas Tecnologias da Informação e Comunicação. Contudo, no contexto da Pandemia do Coronavírus 2019-2020 é preciso repensar as reservas e desafios dos serviços clínicos psicológicos mediados pelas TIC, pois eles se tornaram importantes para uma grande parcela da população brasileira situada no distanciamento social, isolamento domiciliar e/ou em quarentena, contextos que possibilitam o surgimento e/ou potencializam fenômenos como angústia, ansiedade e o estresse.

Palavras-chave: Psicologia. TIC. Pandemia do Coronavírus. Serviços clínicos.

The challenges of psychological services online in 20192020 Coronavirus Pandemic context

\section{Abstract}

In 2019-20 coronavirus pandemic context the clinical psychological services mediated by information and communication technology has become essential to mental health. These services are clinical activities performed by registered psychological at the Braziliam Federal and Regional Psychology Councils through the e-Psi sistem. This article aims critically reflect about technology, viewing the clinical psychological services mediated by information and communication technology in the 2019-2020 coronavirus pandemic context. The qualitative was the approach used, descriptive-comprehensive-interpretative method and authors' interdisciplinary inclination and the collection of data was done from the primary sources through legislative, normative, scientific and informational documents, as well as news published on the Psychology Federal Council's website.The theoretical contribution goes through the Information Science field and is based on the theories of the Information Technology Revolution and Technology's Philosophy. When considering the relationship between technology, Psychology, human behavior and clinical psychological services, the Psychology field goes on useing classic theories to interpret new behavioral and social constructs originated in the Information and Knowledge Society. Between 1995 and 2012 in the historical-epistemic perspective, there were significant advances regarding theoretical, technical and epistemic studies 


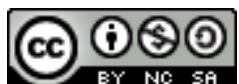

Este trabalho está licenciado com uma Licença Creative Commons - AtribuiçãoNãoComercial-Compartilhalgual 3.0 Brasil. about the clinical psychological services mediated by information and communication technology. However, in the 2019-2020 coronavirus pandemic emerges the needs to rethink reservations and challenges regarding the use of technologies in clinical psychology emerges, because they have become important for a large portion Brazilian population located in social distance, home isolation and / or quarantined, contexts that enable the emergence and / or enhance phenomena such as anguish, anxiety and stress.

Keywords: Psychology. ICT. Coronavirus pandemic. Clinical services.

\section{Introdução}

As tecnologias estão presentes no cotidiano humano, sobretudo, no campo científico, pois, a ciência pode ser uma tecnociência (BACHELARD, 1996; SISSON, WINOGRAD, 2012). Na Psicologia que visualiza as suas reflexões sobre o comportamento, o uso das tecnologias emerge como vetor de sua produção científica, além disso ela é considerada como qualquer artefato ou conjunto de informações que ajudam nas atividades cotidianas humanas.

Partindo deste pressuposto, diversos autores como Skinner (Caixa-Experimental), Pavlov (fístula - cano, conduta, canal), Thorndike (gaiola), Freud (pêndulo) e Wundt, utilizaram da tecnologia como um suporte em seus diversos estudos no âmbito da Psicologia. Porém, este último, em 1985, na cidade de Leipzig, contribui para a autonomia desse campo ao construir seu laboratório de psicologia experimental, marcando assim o racionalismo aplicado na área, onde se nota a abordagem de aproximação entre empirismo e o racionalismo, pois para psicologia não basta raciocinar sobre o comportamento é preciso experimentar (BACHELARD, 1996; CUPANI, 2016; COSENTINO, 2006; DAVIDOFF, 2001; SANTANA; SANTOS; FRANCA, SILVA, 2011).

Na Sociedade da Informação e do Conhecimento (SIC) as Tecnologias de Informação e Comunicação (TIC) são artefatos tecnológicos que marcam todos os espaços sociais, e por consequências, sua construção, acesso e uso fazem emergir novos fenômenos que se intricam na Realidade de Mediação Social e Física, contexto esse, em que o sujeito tem o contato físico, subjetivo e simbólico com as coisas, as pessoas e os fenômenos naturais, químicos e físicos (SANTANA; MARTINS; SILVA, 2016). De tal modo, as análises destas consequências é dever de qualquer campo científico por uma inclinação prática, teórica, epistêmica, interdisciplinar. Porém, na Psicologia não há inclinação epistêmica mais efetiva em relação às consequências, sobretudo, do acesso e uso das TIC pelos psicólogos quanto a produção de psicológica científica e clínica (LEITÃO; NICOLACI-DA-COSTA, 2003).

As autoras Leitão e Nicolaci-Da-Costa (2003) fazem uma crítica pertinente à Psicologia através do texto "Psicologia no novo contexto mundial", revelando que a produção científica na ciência psicológica contínua utilizando as teorias clássicas na interpretação dos impactos ou fenômenos psicológicos e sociais originados na SIC. Para elas os psicólogos analisam novos construtos comportamentais e sociais por teorias que não dão conta dos fenômenos, deixando de buscar o novo que está intrínseco na subjetividade do sujeito na SIC. Vale destacar que a crítica em relação à utilização das teorias clássicas não implica o abandono e/ou rompimento com as mesmas, a sugestão é agregação as teorias clássicas as correntes teóricas contemporâneas como as teorias pós-modernas, as teorias da modernização reflexiva e a teoria da Revolução da Tecnologia da Informação (LEITÃO; NICOLACI-DA-COSTA, 2003).

Assim como excedentes da construção, acesso e uso das TIC emergem a realidade social digital e virtual, onde o sujeito tem contato excessivo, subjetivo e simbólico com os simulacros das coisas e as pessoas. Especialmente no contexto da Pandemia do Coronavírus 2019-20 que tornou essa realidade um contexto seguro, porém, há necessidades que os sujeitos nela sejam amparados quanto a saúde mental por meio dos atendimentos psicológicos on-line.

O objetivo deste artigo é refletir de forma crítica sobre o papel da tecnologia quanto aos atendimentos psicológicos mediados pelas TIC no contexto da Pandemia do Coronavírus 2019-20, assim mentalizando a reconfiguração e/ou rompimento de paradigmas obstáculos do uso das TIC na ciência Psicológica. 


\section{Método e marcha teórico metodológica}

A abordagem qualitativa, com o método descritivo-compreensivo-interpretativo como dominante para uma nova compreensão da relação acesso e uso das TIC na ciência psicológica com o foco na mediação dos serviços clínicos psicológicos (MASINI, 2004). A inclinação interdisciplinar de autores ao qual se refere Teixeira (2004) e Carlos (2007), visualiza a interdisciplinaridade que é imperativa nos estudos em Psicologia, pois nesta ciência os estudos das TIC se encaixam na perspectiva de temas transversais.

A coleta de dados ocorreu de fontes primárias por meio de documentos legislativos, que incluem as resoluções que orientam os psicológicos quanto os serviços psicológicos mediados pelas TIC; documentos normativos, que incluem o código de Ética do Psicológico e cartinhas; documentos científicos, que agregam artigos, dissertações e teses que tratam da temática, e informativos, que se refere as notícias publicadas no site do Conselho Federal de Psicologia.

O aporte teórico da Ciência da Informação se apoiou nas teorias da Revolução da Tecnologia da Informação que ancora as discussões sobre os processos que permeia as subjetividades e identidades pela cultura do acesso e uso das TIC e da informação. Quanto a Filosofia da Tecnologia, ela visualiza os três pilares básicos: O que é a tecnologia para a Psicologia? Como os sujeitos agem frente a elas? E como a psicologia deve agir sobre os reflexos da relação sujeitos e TIC, visualizando os serviços psicológicos mediados pelas TIC? (CUPANI, 2016; COSENTINO, 2006).

O artigo é um retorno aos serviços psicológicos mediados pelas TIC, seu surgimento, desenvolvimento, vantagens, desvantagens, utilização e, especialmente, os desafios destes serviços no contexto da Pandemia do Coronavírus 2019-20 na SIC. Pois este retorno possibilita a reconfiguração de paradigmas marcantes em Bachelard (1996) assim revendo as possibilidades destes serviços, como no rompimento semelhante em Kuhn (1987), que se refere às falhas de pesquisas sobre a temática, ao qual ambas as concepções visualizam a retomada dos estudos para os avanços da psicoterapia e orientação on-line na ciência psicológica.

\section{Aspectos histórico-epistêmicos dos serviços psicológicos mediados pelas TIC}

Antes mesmo da popularização das TIC, há apenas 50 anos o aconselhamento psicológico encontrava na tecnologia (gravações telefônicas e áudio, entre outras) uma grande aliada em que a cyberterapia, ou terapia on-line é também visualizada (LANDA DURÁN; PEÑALOSA CASTRO, 2009). Contudo, é a partir da década de 1990 que ocorre o interesse crescente da utilização das TIC na psicologia clínica. Os primeiros atendimentos no contexto nacional foram oferecidos ainda em 1995 pelos endereços eletrônicos: Help on-line e Psicólogos on-line. Ainda que timidamente e sem o respaldo da American Psychological Association (APA) e do Conselho Federal de Psicologia (CFP) (CAMARGO, 1999).

Neste período este tipo de serviço causou uma tensão, pois observou-se à falta de ética destes profissionais em que o próprio CFP por meio da porta voz, ocasionando a acusação dos primeiros psicólogos brasileiros de má-fé pela Presidente do Conselho, Ana Bock. A partir disto, nota-se primeiramente que a atividade se configurava ainda ilegal no país, e segundo, não existia nenhuma comprovação científica acerca da eficácia da psicoterapia e orientação on-line. E por último, o fato dos serviços serem cobrados (CAMARGO, 1999).

Estes primeiros atendimentos, ainda que na clandestinidade, já apresentavam características dos dois serviços pautados pela Resolução no 003/2000. Assim, por caminhos tortuosos, já se configurava a necessidade de reavaliação das atividades dos psicólogos clínicos, ou seja, a influência das TIC começava a refletir nas atividades clínicas psicológicas, gerando a necessidade de, no Brasil, se adequar à realidade social e tecnológica, as quais evidencia os aspectos histórico-epistêmicos (Figura 1, esferas coloridas) dos serviços psicológicos mediados pelas TIC. 
Figura 1- Aspectos histórico-epistêmicos dos serviços psicológicos mediados pelas TIC.

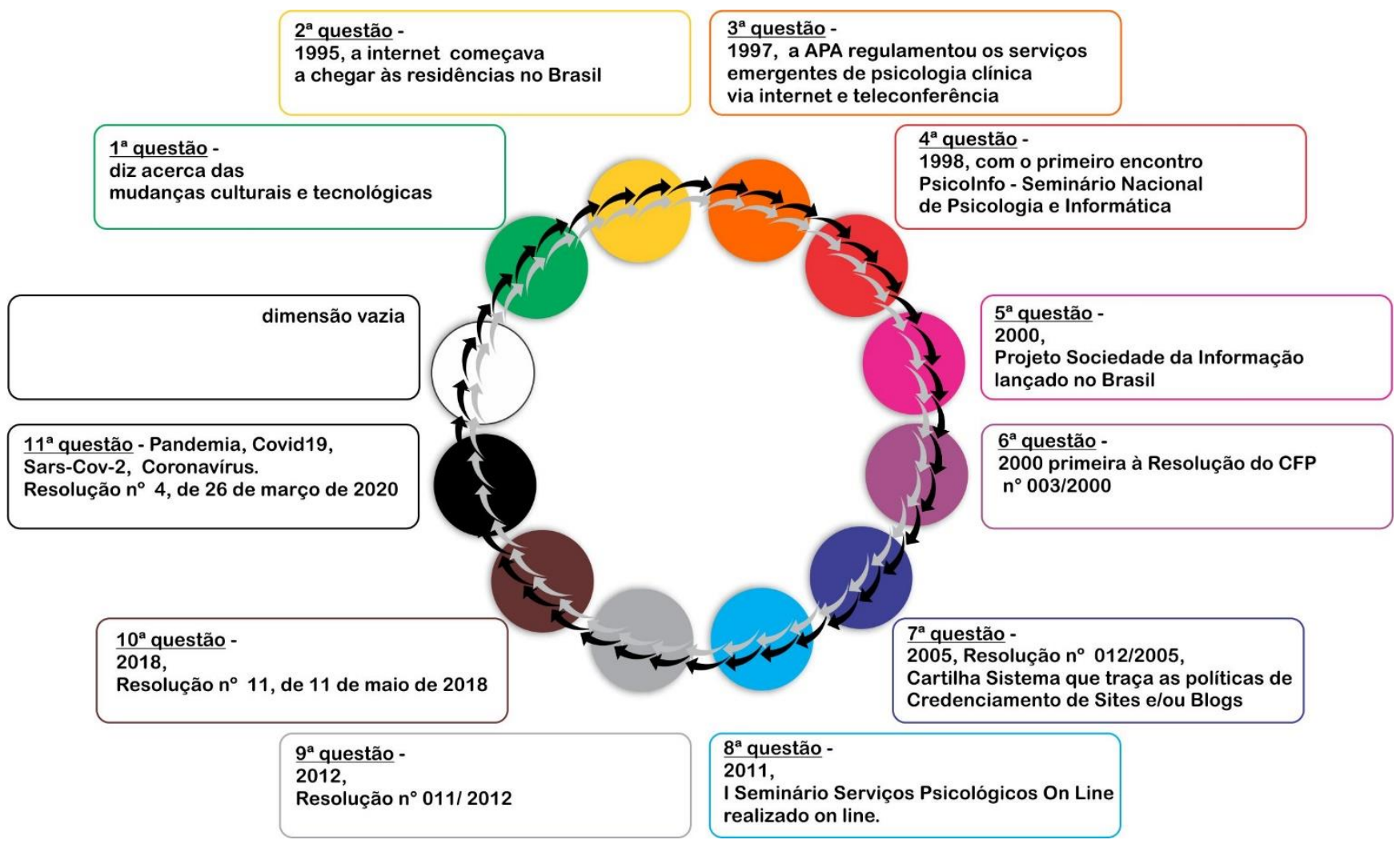

Fonte: Elaborada pelos autores.

A primeira questão (Figura 1, esfera verde) aborda as mudanças culturais e tecnológicas no Brasil e no mundo. A segunda questão (Figura 1, esfera amarela) ocorreu em 1995, quando a internet ainda começava a chegar às residências no Brasil, ano que foi aberto o Núcleo de Pesquisas da Psicologia em Informática (NPPI) da Pontifícia Universidade Católica de São Paulo (PUC-SP) liderado pela psicóloga Rosa Farah. A priori o objetivo do grupo era a compressão do fenômeno da internet, contudo quando o site do NPPI foi ao ar, uma demanda emergiu, onde muitos sujeitos começaram a mandar emails pedindo ajuda psicológica. E de 1997 a 2009 esse número de e-mails aumentou consideravelmente. Porém, a demanda não era considerada pelo CFP que não se preocupou com o fenômeno (ATENDIMENTO..., 2011).

A terceira questão (Figura 1, esfera laranja) ocorreu em 1997 quando a APA regulamentou os serviços emergentes de psicologia clínica via internet e teleconferência (RODRIGUES, 2014). A quarta questão (Figura 1, esfera vermelha) se dá em 1998, com o primeiro encontro Psicolnfo - Seminário Nacional de Psicologia e Informática, que continuará no ano seguinte, no centro de um grupo denominado Trabalho sobre Atendimento mediado pelo Computador, instituído por proposta do próprio conselho, evento que teve edições em 2003 - com o II Psicolnfo, e em 2006 com o III Psicolnfo.

A quinta questão (Figura 1, esfera rosa) se dá por meio do Projeto Sociedade da Informação lançado em 2000 no país, com os ideais de modernização, pois o projeto SI traça as diretrizes para alcançar as metas arroladas à construção de uma unidade nacional "sociedade mais justa" como também a preservação de nossa identidade cultural, desenvolvimento sustentável e o equilíbrio regional e a efetiva participação social para uma democracia política. O Livro Verde: Ciência Tecnologia e Inovação - delineia as criações e orientações das políticas de ajuste da sociedade brasileira ao contexto das TIC para constituir a institucionalidade necessária para enfrentar o desafio da inovação, elemento determinante das políticas públicas em todo o mundo, que deve ser criativamente enfrentado pela sociedade brasileira. Ou seja, texto onde contem a criação das políticas, estratégias de ajustes do Brasil ao contexto das TIC (TAKAHASHI, 2000). 
A sexta questão (Figura 1, esfera roxa) também acontece em 2000 e diz respeito da primeira à Resolução do n 003/2000, fruto de debates no encontro do Psicolnfo - Seminário Nacional de Psicologia e Informática em 98. Então, a partir deste ano foi aprovado, depois de intensas articulações e discussões com os representantes dos CRPs de todo o contexto nacional, realizadas em plenárias, grupos de trabalho, simpósios e seminários. Torna-se fato a resolução $n^{\circ}$ 003/2000 que regulamenta o atendimento psicoterapêutico e outros serviços psicológicos mediados pelas TIC. Em suma, é regulamentado o atendimento psicoterapêutico on-line e outros serviços psicológicos mediados pelas TIC e que em outros contextos são chamados de psyberterapia, psyberpsicoterapia, psyberatendimento, cyberterapia, cyberpsicoterapia, cyberatendimento, eterapia, webpsicoterapia, webpsicanálise (BRASIL, 2000).

A sétima questão (Figura 1, esfera azul escuro) diz acerca da Resolução no 012/2005, junto com o lançamento da Cartilha Sistema que traça as políticas de Credenciamento de sites e/ou blog, ao qual informa todos os passos de cadastramento e análise antes de receber o Selo Certificador que era emitido pelo CFP permitindo o psicólogo usar estes instrumentos tecnológicos (BRASIL, 2005a; BRASIL, 2005c).

A oitava questão (Figura 1, esfera azul claro) se dá em 2011 com o primeiro Seminário Serviços Psicológicos on-line realizado virtualmente em 22 de novembro de 2011 transmitido pelo site do CFP. Evento específico que discutiu a temática e os aspectos interdisciplinares presentes nos serviços psicológicos on-line, como reza a Resolução ํㅜ 12/2005. Assim, por meio das conferências as "Diversas formas da psicologia: psicoterapia on-line" por Rosa Farah e "Cibercultura, novas mídias e produção de subjetividade" por José Carlos Ribeiro. Como as mesas de "Psicoterapia on-line: histórico no Brasil e no mundo por Luciana Ruffo e Maria Adelia Pieta (PIETA, 2011).

E a nona questão (Figura 1, esfera cinza) diz acerca da Resolução nº 011/ 2012 que substituiu a Resolução n.․ 12/2005, sendo substituído pela Resolução no 11/2018, junto com e-Psi um sistema lista dos psicólogos autorizados pelo Sistema Conselhos de Psicologia a prestarem serviços psicológicos mediados pelas TIC, que represa a décima questão (Figura 1, esfera marrom), que é uma reconfiguração do Selo Certificador que fora utilizado a partir de 2005.

A décima primeira questão (Figura 1, esfera preta) diz sobre o contexto da Pandemia do Coronavírus 2019-20, explicada no tópico 3.1. Quanto à esfera branca da Figura 1 intitulada de vazia, representa a ideia de que os estudos sobre os serviços psicológicos mediados pelas TIC devem se manter sempre atualizados, pois sempre haverá novos fenômenos a serem descobertos e debatidos sobre a temática no âmbito teórico-epistêmico (Figura 1, círculo preto de setas) e técnicoepistêmico (Figura 1, círculo cinza de setas).

\subsection{O contexto da Pandemia do Coronavírus 2019-20 e a dimensão da angústia, ansiedade e estresse}

Considerando o fluxo dos aspectos histórico-epistêmicos dos serviços psicológicos mediados pelas TIC (Figura 1) o contexto da Pandemia do Coronavírus 2019-20 se configura a décima primeira questão, sendo imperativo entendê-la antes de figurá-la como um possível vetor epistêmico. 
Figura 2 - Contexto da Pandemia 2019-20 e suas dimensões.

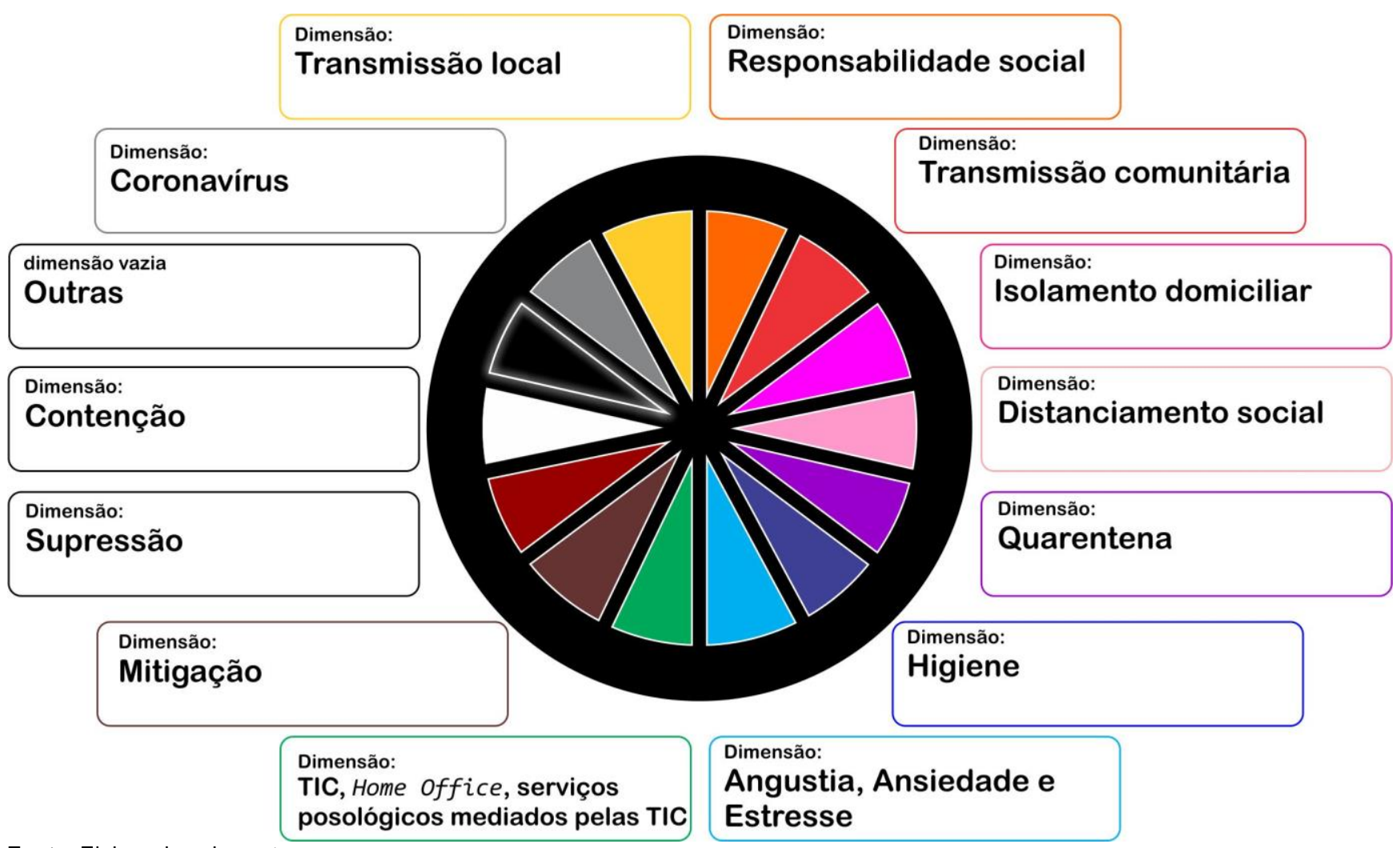

Fonte: Elaborada pelos autores.

Uma Pandemia (Figura 2, esfera preta com dimensões coloridas) é caracterizada como uma epidemia de larga distribuição geográfica, atingindo mais de um país ou continente (BRASIL, 2020f; RIBEIRO,2020). Neste sentido, o uso da expressão Pandemia do Coronavírus 2019-20, pode ser empregado, pois já ultrapassa a casa de milhares de vítimas em todo o mundo. O termo coronavírus (Figura 2, dimensão cinza) refere-se a grande família de vírus que pode causar desde resfriados comuns até doenças respiratórias mais graves, como a Síndrome Respiratória Aguda Grave (SARS) e a Síndrome Respiratória do Oriente Médio (MERS) (UNIVERSIDADE FEDERAL DO CARIRI, 2020).

Assim, o novo coronavírus foi descoberto em dezembro de 2019 na China, que se deu a partir dela a Transmissão local (Figura 2, dimensão amarela), essa que se refere contaminação de sujeito no exterior, ao qual mais tarde ele traz o vírus para outros países, e o transmite para os sujeitos de seu convívio, configurando nestes casos esse sujeito/paciente como um foco da infecção.

Diante de uma pandemia, emergem as Responsabilidade Social (Figura 2, dimensão laranja) como dimensão estratégica, visualizando-a pela empatia, da ética, moral e responsabilidade (TARGINO, SANTANA; GARCIA; SOUZA, 2019), quando se refere aos sujeitos que têm consciência pela Transmissão local (Figura 2, dimensão amarela) e comunitária (Figura 2, dimensão vermelha), como o papel das instituições, governantes frente as informações sobre a disseminação do virus.

A Transmissão comunitária (Figura 2, dimensão vermelha) se refere o contato de sujeitos sem veículo com casos confirmado, em uma determinada área onde não é possível rastrear a origem de cada infecção. O Isolamento domiciliar (Figura 2, dimensão rosa), se refere ao sujeito que testou positivo para o Coronavírus, onde a reclusão se dá sobre aqueles 
que estiveram em contato com pessoas contaminadas pelo vírus e estão aguardando para ver se apresentam os sintomas (BRASIL, 2020f; UNIVERSIDADE FEDERAL DO CARIRI, 2020; RIBEIRO,2020).

Como estratégia de contenção emerge o distanciamento social (Figura 2, dimensão rosa claro), prática utilizada pelos sujeitos que estão fazendo a sua parte para reduzir o risco da disseminação, sua saída de casa refere as necessidades básicas como: comprar alimentos, remédios e atendimento médico (UNIVERSIDADE FEDERAL DO CARIRI, 2020). Contudo, independentemente, entre sujeitos infectados e não infectados, a Higiene (Figura 2, dimensão azul), sobretudo, das mãos torna-se uma estratégia importante neste contexto.

A Quarentena (Figura 2, dimensão roxa), é um ato administrativo, ao qual o Ministério da Saúde regulamentou seus o critérios que devem ser aplicados pelas autoridades de saúde local para sujeitos com suspeita e/ou a confirmação de infecção (UNIVERSIDADE FEDERAL DO CARIRI, 2020; RIBEIRO, 2020).

Uma vez havendo sobretudo, o isolamento domiciliar (Figura 2, dimensão rosa), quarentena (Figura 2, dimensão roxa),e o distanciamento social (Figura 2, dimensão rosa claro), pode ocorrer os fenômenos como a Angústia, Ansiedade e Estresse (Figura 2, dimensão azul claro), que de forma didática chamaremos neste artigo de AAE Covid-19, que é o imbricamento ou não dos fenômenos psicológicos que emergem dos vetores que constituem o contexto da Pandemia do Coronavírus 201920.

Na dimensão acesso e uso das TIC (Figura 2, dimensão verde), destaca-se o Home Office que por sua vez possiblita os serviços posológicos mediados pelas TIC, que se compreende como um conjunto de artefatos e conjunto de informações epistêmicas, teóricos e técnicos que integram entre si por meio de estruturas materiais, funcionais, lógicas e simbólicas que agregam hardware, software e telecomunicações, que inclui também o computador, a internet, e hipertexto, entre outras (SANTANA, 2016). E dentro do acesso e uso das TIC, destca-se o Home Office, todo trabalho que se encaixa nesta modalidade, pois a prestação de serviço ocorre predominantemente fora das dependências da empresa, podendo eventualmente se por um período longo, por contrato e/ou de forma emergencial, como no caso de enchentes, greves de transporte público ou prevenção de epidemias ou pandemias, que nestes casos o Home Office pode ocorrer a priori sem o formalismo legal (GRANATO, 2020).

Neste sentido visualizando as TIC e o Home Offce no contexto da Pandemia do Coronavírus 2019-20 os serviços posológicos mediados pelas TIC ocorrem pela conexão do acesso à internet por hardwere e software, websites, aplicativos, plataformas digitais dispositivos móveis, TV e aparelhos conjugados ou híbridos e qualquer outro modo de promoção da interação de maneira síncrona (chat, conferências) ou assíncrona (e-mails, mensagens) que possa vir a ser implementado e que atenda ao objeto da Resolução no 11/2018 (BRASIL, 2018; PONTIFÍCIA UNIVERSIDADE CATÓLICA, [2018]).

Além disso, há também a Mitigação (Figura 2, dimensão marron) que é a perda do rasteio da epidemia, ou seja, quando constata a transmissão comunitária, assim surge a necessidade de medidas para combater a evolução rápida de novos casos evitando o colapso do Sistema de Saúde, buscando proteger sujeitos vulneráveis e casos graves. Além da Supressão (Figura 2, dimensão vinho), estratégias para romper as cadeias de transmissão, como isolamento social e outros como a Contenção (Figura 2, dimensão branca), medidas adotadas no início de uma epidemia para evitar o contágio de mais pessoas, como rastreamento e isolamento (UNIVERSIDADE FEDERAL DO CARIRI, 2020).

Neste sentido, diante do contexto da Pandemia do Coronavírus 2019-20 e suas dimensões há uma reorganização do mundo, ao qual diz também acerca dos aspectos tecnológicos e técnicos que mobiliza as confrarias científicas em todo o mundo quanto a um antídoto do vírus em si, como também reflexos de ordem social, econômico, e psicológica, ou seja, sobre a saúde mental. Diante deste avanço, na terça-feira dia 24 de março de 2020, CFP enviou recomendação, para gestores públicos e empregadores de psicólogos que suspendessem de forma imediata as atividades profissionais da categoria na modalidade presencial, salve as exceções emergenciais (BRASIL, 2020e, 2020a, 2020b, 2020c, 2020d).

Neste sentido, se tornou imperativo considerar a AAE Covid-10 (Figura 2, dimensão verde) do contexto da Pandemia do Coronavírus 2019-20, disponibilizando os serviços psicológicos mediado pelas TIC, em que a urgência sensibilizou o CFP 
que possibilitou que Psicólogos prestassem atendimento à distância sem a aprovação prévia do cadastro e-Psi de forma voluntária e ou paga, assistida por planos de saúde. Porém, o CFP sinalizou a obrigatoriedade da realização do cadastro no e-psi.cfp.orb.br. Ao qual flexibilização de iniciar os atendimentos antes do seu cadastro e suas análises pelo seu Conselho Regional cobrindo apenas os meses de março e abril (BRASIL. 2020e. 2020a, 2020b, 2020c, 2020d).

Contudo, diante do AAE Covid-10 (Figura 2, dimensão azul claro), do contexto da Pandemia do Coronavírus 2019-20, faz com que os conselhos de psicologia repensarem também pela Responsabilidade social pelo caminho da ética pelo auxílio da Psicanálise do conhecimento científico quanto as estratégias epistêmicas para compreender e minimizar as forças dos aspectos axiológicas que perpassam seus interesses, as intenções e as comodidades. Visualizando uma Psicologia em que os estudiosos desapeguem das suas lanternas do conhecimento, que se figura um obstáculo epistemológico para o avanço científico da psicologia quanto às TIC no âmbito clínico (TARGINO, SANTANA; GARCIA; SOUZA, 2019).

Isso significa minimizar as preocupações, reservas e desconfianças quanto a utilização das TIC (Figura 2, dimensão verde) e as inferências sobretudo, negativas de seu acesso e uso (COSENTINO, 2006; SANTANA; MARTINS; SILVA, 2016), pois as mesmas vê contribuindo significantemente no contexto da Pandemia do Coronavírus 2019-20, tornando-se o diferencial, por sua possibilidade de comunicação e contato entre os sujeitos originando imperativos que mostram sua dimensão na ciência Psicológica, pelos psicólogos e instituições como os Conselhos de Psicologia, visualizando o empenho de pesquisas, eventos, debates, qualificação para que os serviços posológicos mediados pelas TIC se tornem uma prática cotidiana, efetiva e de qualidade. Contudo, neste cenário da Pandemia do Coronavírus 2019-20 emergem como fenômeno de repensar as reservas e desconfianças quanto o uso das TIC na psicologia clínica.

\section{Postura dos Conselhos de Psicologia e dos Psicólogos quanto às TIC}

A utilização das TIC pelos profissionais da Psicologia exige a reflexão sobre três questões que se complementam e que estão pautadas na primeira Resolução no 003/2000, e perpetuada pela Resolução no 012/2005, no 011/2012 e atualizada pela resolução oํ 11/ 2018,

[...] é dever da (o) psicóloga (o) prestar serviços psicológicos de qualidade, em condições de trabalho dignas e apropriadas à natureza desses serviços, utilizando princípios, conhecimentos e técnicas reconhecidamente fundamentados na ciência psicológica, na ética e na legislação profissional (BRASIL, $2000,2005 c, 2012,2018)$

A primeira questão é de fundo teórico, ao campo epistêmico (conhecimentos), pois o psicólogo deve estar inteirado acerca das teorias contemporâneas que giram em torno das tecnologias, assim construindo uma discussão interdisciplinar de autores, áreas, teorias e técnicas quanto à relação TIC e Psicologia para compressão da filosofia da informação, dos fluxos comunicacionais e informacionais e curadoria de dados e da informação (LEITÃO; NICOLACI-DA-COSTA, 2003; TEIXEIRA, 2004; CARLOS, 2007).

A segunda refere-se a técnica (técnicas), ao entender básico do uso destes instrumentos tecnológicos como também das terminologias e linguagens que alinham internet, ciência e os fluxos informacionais da dimensão de bits para a compressão do teor ambivalente e ambíguo da realidade social digital e virtual como o seu imbricamento com as duas facetas que compõe a SIC (NEGROPONTE,1995; DEMO, 2000; RUFFO, 2011, BRASIL, 2013; BRASIL, 2018).

A terceira questão diz sobre a ética, pois o CFP reforça a possibilidade de exercício profissional na atuação em emergências, em contextos clínicos e Assistência Social e de políticas públicas. Nos casos em que os psicólogos optem pela prestação de serviços psicológicos realizados por meios das TIC, deverá seguir as orientações da Resolução ํㅡ 4, de

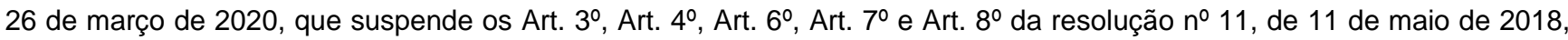
em especial da necessidade de realização de um cadastro prévio junto ao seu Conselho Regional, pois a flexibilização de iniciar os atendimentos por meio das tecnologias deve ocorrer antes do seu cadastro para ser analisado pelo Conselho, já que está prevista apenas para os meses de março e abril de 2020 (BRASIL, 2018; BRASIL, 2020f).

Diante destas informações é relevante frisar um interesse por parte do psicólogo, sobretudo, dos Conselhos Regionais e Federais de Psicologia para promoção da formação dos psicólogos quanto estudos das tecnologias. Pois resoluções se figuram a condição primeira, já que faz-se necessário ir além das ações, orientações, recomendações e normativas, além de 
visualizar a formação continuada dos profissionais frente as TIC no sentido de desenvolver estes serviços com eficiência, eficácia, qualidade, e, sobretudo, promover estratégias de alcance para mais grupos sociais.

\section{Modalidades dos serviços psicológicos mediados pelas TIC}

As modalidades dos serviços psicológicos mediados pelas TIC são: a consulta, psicoterapia, orientação (psicoterapia breve focal), processos de seleção de pessoal, supervisão técnica e aplicação de testes psicológicos, desde que devidamente autorizados pelo Sistema de Avaliação de Testes Psicológicos (SATEPSI) e normativas vigentes do CFP. Assim, se compreendem por esses serviços as atividades clínicas realizadas por psicólogos cadastros nos Conselhos Regionais no sistema no e-Psi, que utilizam as TIC (BRASIL, 2018; PONTIFÍCIA UNIVERSIDADE CATÓLICA, [2018]).

Antes da Resolução no 11/2018 era mais didático fazer uma destinação entre psicoterapia, orientação (psicoterapia breve), estes que compõem os serviços psicológicos on-line o que não especificado na atual resolução. De tal maneira, o CFP por meio da Resolução no 11/2018, apenas regulamenta a prestação de serviços psicológicos realizados por meios da TIC e revoga a Resolução n.․ 11/2012. Fortim e Cosentino (2007) descrevem uma distinção entre a psicoterapia on-line e orientação psicológica on-line. A psicoterapia on-line diz respeito a um modelo de psicoterapia presencial adaptado ao contexto da internet. Ao qual naquela época os CFP junto com os Conselhos Regionais e os Serviços Psicológicos terapêuticos on-line ainda estão em caráter experimental, pois eram somente oferecidos quando o psicólogo executava mediante um projeto de pesquisa para fins ivestigativos.

[...] com protocolo de pesquisa aprovado por Comitê de Ética em pesquisa reconhecido pelo Conselho Nacional de Saúde, conforme Resolução CNS 196/96 ou legislação que venha a substituí-la. Deve respeito às Resoluções específicas do Conselho Federal de Psicologia para pesquisas com seres humanos em Psicologia e ao Código de Ética Profissional do Psicólogo [...] (BRASIL, 2005c, p.3).

Tendo o caráter experimental como investigativo não podiam ser cobrados como um processo de psicotepia tradicional. $O$ conceito mais atualizado de psocterapia on-line e de Rodrigues $(2014$, p.22) psicoterapia online, vista como uma modalidade de atendimento psicológico "[...] individual realizado por meio da internet, de modo sistemático e contínuo, semelhante aos atendimentos clínicos face a face que ocorrem em consultório por um psicoterapeuta registrado no CRP".

No que tange a orientação on-line, essa trabalha com limites muito delicados entre o espaço terapêutico e a psicoterapia, pois não se constrói um elo entre o cliente e o psicólogo pois o processo se resume entre oito e doze encontros virtuais por Skype e/ou e-mails trocados. Em suma, deve haver um limite de troca de informações entre o psicólogo e o cliente e que ganhou a abordagem de psicoterapia breve on-line que não é indicada para todos os casos. Segundo o Pontificia Universidade Católica (2020) ela não é indicada para sujeitos que apresentem transtornos psicóticos e/ou que tenham algum tipo de risco com relação ao suicídio (FORTIM; COSENTINO, 2007; RODRIGUES, 2014; PONTIFÍCIA UNIVERSIDADE CATÓLICA, [2018]).

De acordo com as resoluções de 2000, 2005 e 2012 os conteúdos destes encontros faziam referências às questões breves, objetivas, pontuais e informativos centradas nos assuntos específicos como:

[...] afetivo-sexual, [...] orientação profissional, orientação de aprendizagem e Psicologia escolar, orientação ergonômica, consultorias a empresas, reabilitação cognitiva, ideomotora e comunicativa, processos prévios de seleção de pessoal, utilização de testes informatizados devidamente validados, utilização de softwares informativos e educativos com resposta automatizada [...] (BRASIL, 2000, 2005c).

Portanto, estes serviços podiam ser realizados desde que não ferissem o Código de Ética Profissional do Psicólogo e as resoluções do CFP de 2000/2005 e 2012. Igualmente, o método buscava compreender a questão central na queixa específica relatada pelo cliente, para posteriormente se fazer a orientação pontualmente. Porém, na Resolução oㅜ 11/2018, apenas se autorizam dos serviços psicológicos realizados por meios das TIC (BRASIL, 2005c.; BRASIL, 2018).

A utilização das TIC pode reduzir cada vez mais os serviços de psicoterapia e orientação on-line à população geral, visto que o serviço de psicoterapia tradicional já se configura um bem simbólico das classes mais privilegiadas, pois na SI a 
desigualdade social que atravessa também permeia na Realidade de Mediação Social e Física. Mesmo com o projeto de SI a utilização das TIC no Brasil ainda é restrita para sujeitos de menor poder aquisitivo (COSENTINO, 2006).

Uma desvantagem que pode ser questionada diz respeito ao registro de dados que emerge por meio da linguagem do corpo fornecida pelo cliente. Elementos que são compartilhadas das mais diversas formas durante a sessão psiterapêutica tradicional revelam muitas questões acerca do cliente. Para minimizar esta desvantagem que acontece quando os sentimentos, expressões, raivas, angústias, alegrias, entre outras não podem ser captados, podem ser usados os emoticons que são formas visuais de uma comunicação para linguística, às vezes denominada de Smiley, que os símbolos que frequentemente se utiliza em conversas on-line. Marot (2003) revela que se o método feito por meio da tecnologia de videoconferência (híbrida) que possibilitar tanto o contato visual, aliado a troca de dados da linguagem verbal e não verbal, a problemática se desfaz.

Quanto as vantagens, os usuários em potencial destes serviços serão sujeitos de cidades pequenas que não tem clínicas, imigrantes e refugiados, surdos e mudos, e, sobretudo, uma parcela da população em contexto de epidemias e pandemias, às quais estas duas últimas citadas agregam todos as situações citadas, visto que uma pandemia é um fenômeno maior. Contudo, uma vez ocorrendo a superação de uma pandemia, as situações das cidades pequenas, dos imigrantes e refugiados, como os casos dos surdos e mudos ainda demandará os serviços psicológicos mediados pelas TIC.

\section{Considerações Finais}

A ciência psicológica visualiza o comportamento como objeto de estudo, sendo compressivo que o foco nas tecnologias e nos reflexos delas não estejam situados no escopo de seu projeto disciplinar de ciência. Contudo, visualizando a SIC como um contexto das ambiguidades e ambivalências que emergem através dos fluxos informacionais e comunicacionais, se torna pertinente uma inclinação da ciência psicológica para lógica epistêmica interdisciplinar, uma vez que é de sua competência os serviços psicológicos mediados pelas TIC. Assim, marchar pela interdisciplinaridade pode oferecer a Psicologia as condições de situar os estudos das tecnologias no seu âmbito como temática transversal para entender como os sujeitos se comportam frente a elas, com delimitar o papel da Psicologia na análise dos reflexos da relação sujeitos e TIC e o papel da tecnologia na sua na clínica e na produção cientifica como vetor epistêmico.

No contexto da pandemia do coronavírus 2019-20, os serviços clínicos psicológicos mediados pelas TIC se tornaram importantes no que versa as demandas da população brasileira que se encontra situada na realidade social digital e virtual, por meio do distanciamento social (fisico), isolamento domiciliar e/ou em quarentena, fatores que também potencializam, como dão contornos mais definidos a angústia, a ansiedade e o estresse como construtos que também ameaçam a boa saúde mental. Esses serviços se configuram também estratégicos para resguardar os profissionais psicólogos que podem manter seus atendimentos pela lógica do Home Office. Contudo, assistir à população a partir deste modelo clínico é desafiador, pois há fatores a se considerar que emergem sobretudo pela ética, a formação teórica e técnica dos psicólogos e uma delimitação mais conceitual terminológica, e outras especificidades que compõe cada modalidade que faz parte dos serviços psicológicos mediados pelas TIC. Quanto a vantagem, esse método é fundamental para manter a calma e um estado emocional apropriado.

Uma população bem informada pode atuar de modo adequado, proteger-se melhor e ser menos vulnerável do ponto de vista psicossocial. O uso das TIC possibilitou mudanças benéficas em meio a essa pandemia, fazendo com que a maioria dos profissionais se adequassem utilizando os meios digitais como a principal ferramenta de serviço.

Ao fazer uma análise a partir de uma perspectiva histórica-epistêmico, infere-se que entre 1995 a 2012 ouve avanços significativos acerca dos estudos teórico-epistêmicos e técnico-epistêmicos sobre os serviços psicológicos mediados pelas TIC. Contudo, se faz compreender que o contexto da pandemia do coronavírus 2019-20 pode ser fortuito para o progresso da psicologia quanto a estes serviços, cabendo aos conselhos de psicologia pensar na construção de protocolos para coletas de dados dos atendimentos que estão ocorrendo, pois, é também no contexto de uma crise que se há crescimento.

Esse contexto pode-se considerar um vetor epistêmico, pois ele tem reestruturado o mundo, o que inclui o campo cientifico ao qual o Brasil tem sofrido perdas pela gestão do atual presidente e ministro da educação. Em um primeiro momento as 
reconfigurações no âmbito da psicologia deu-se pela urgência da facilitação dos psicológicos quanto aos atendimentos a população sem cadastro e análise prévio no e-Psi, embora, o CFP deixou claro a urgência do cadastro. Houve também a

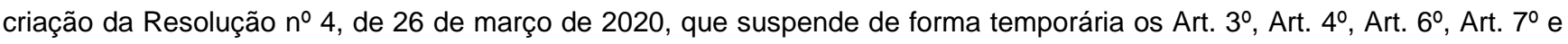
Art. $8^{\circ}$ da resolução ํำ 11, de 11 de maio de 2018 que dificultavam a aplicação dos serviços clínicos em contextos estremos.

\section{Referências}

ATENDIMENTO psicológico na internet começou em 1997. Folha de São Paulo, São Paulo, 03 jun. 2011. Disponível em: https://www1.folha.uol.com.br/equilibrioesaude/2011/06/924873-atendimento-psicologico-na-internet-comecou-em-1997.shtml. Acesso em: 20 dez. 2019.

BACHELARD, Gaston. A formação do espírito científico: contribuição para uma psicanálise do conhecimento. Rio de Janeiro: Contraponto, 1996.

BRASIL. Conselho Federal de Psicologia. Cartilha Sistema de Credenciamento de Sites Serviços Psicológicos Online: nova ferramenta de credenciamento. 2005a. Disponível em: https://cadastrosite.cfp.org.br/docs/cartilha.pdf. Acesso em: 24 mar. 2020.

BRASIL. Conselho Federal de Psicologia. Código de Ética do Psicólogo. Brasília, DF, 2005b. Disponível em: https://site.cfp.org.br/wpcontent/uploads/2012/07/codigo-de-etica-psicologia.pdf. Acesso em: 19 jun. 2011.

BRASIL. Conselho Federal de Psicologia. Coronavírus: CFP realiza Diálogo Digital sobre atuação profissional no SUS e SUAS. 2020a. Disponível em: https://site.cfp.org.br/coronavirus-cfp-realiza-dialogo-digital-sobre-atuacao-profissional-no-sus-e-suas/. Acesso em: 25 mar. 2020.

BRASIL. Conselho Federal de Psicologia. Coronavírus: CFP recomenda suspensão imediata das atividades presenciais realizadas por psicólogas(os). 2020b. Disponível em: https://site.cfp.org.br/coronavirus-cfp-recomenda-suspensao-imediata-das-atividades-presenciaisrealizadas-por-psicologasos/. Acesso em: 26 mar. 2020.

BRASIL. Conselho Federal de Psicologia. Coronavírus: CFP solicita inclusão de serviços psicológicos on-line na cobertura dos planos de saúde. 2020c. Disponível em: https://site.cfp.org.br/coronavirus-cfp-solicita-inclusao-de-servicos-psicologicos-on-line-na-cobertura-dosplanos-de-saude/ Acesso em: 26 mar. 2020.

BRASIL. Conselho Federal de Psicologia. Coronavírus: Comunicado sobre atendimento on-line. 2020d. Disponível em: https://site.cfp.org.br/coronavirus-comunicado-sobre-atendimento-on-line/. Acesso em: 26 mar. 2020.

BRASIL. Conselho Federal de Psicologia. Nota orientativa às (aos) psicólogas (os): trabalho voluntário e publicidade em Psicologia, diante do Coronavírus (COVID-19). 2020e. Disponível em: https://site.cfp.org.br/nota-orientativa-asaos-psicologasos-trabalho-voluntario-epublicidade-em-psicologia-diante-do-coronavirus-covid-19/. Acesso em: 25 mar. 2020.

BRASIL. Conselho Federal de Psicologia. Resolução no 003/2000 - Regulamenta o atendimento psicoterapêutico e outros serviços psicológicos mediados por computador e revoga a Resolução CFP n 003/2000. 2000.

Disponível em: https://site.cfp.org.br/wp-content/uploads/2000/12/resolucao2000 6.pdf. Acesso em: 30 mar. 2020.

BRASIL. Conselho Federal de Psicologia. Resolução no 011/2012 - Regulamenta o atendimento psicoterapêutico e outros serviços psicológicos mediados por computador e revoga a Resolução CFP n 012/2005. 2012.

Disponível em: https://site.cfp.org.br/wp-content/uploads/2012/07/Resoluxo CFP nx 011-12.pdf. Acesso em: 25 mar. 2020.

BRASIL. Conselho Federal de Psicologia. Resolução no 11, de 11 de maio de 2018 - Regulamenta o atendimento psicoterapêutico e outros serviços psicológicos mediados por computador e revoga a Resolução CFP no 011/2012. 2018.

Disponível em: https://site.cfp.org.br/wp-content/uploads/2018/05/RESOLU\%C3\%87\%C3\%830-N\%C2\%BA-11-DE-11-DE-MAIO-DE2018.pdf. Acesso em: 25 mar. 2020

BRASIL. Conselho Federal de Psicologia. Resolução no 012/2005 - Regulamenta o atendimento psicoterapêutico e outros serviços psicológicos mediados por computador e revoga a Resolução CFP CFP n 003/2000. 2005c.

.Disponível em: http://site.cfp.org.br/wp-content/uploads/2005/08/resolucao2005 12.pdf. Acesso em: 25 mar. 2020.

BRASIL. Conselho Federal de Psicologia. Resolução no 4, de 26 de março de 2020 - Regulamenta o atendimento psicoterapêutico e outros serviços psicológicos mediados por computador e revoga a Resolução no 11, de 11 de maio de 2018. 2020e.

Disponível em: https://atosoficiais.com.br/cfp/resolucao-do-exercicio-profissional-n-4-2020-dispoe-sobre-regulamentacao-de-servicospsicologicos-prestados-por-meio-de-tecnologia-da-informacao-e-da-comunicacao-durante-a-pandemia-do-covid19? origin=instituicao\&q=004/2020. Acesso em: 31 mar. 2020.

CAMARGO, D. Assim é demais! Psicólogos ganham dinheiro oferecendo consultas de até 100 reais pela internet. Veja, v. 1589, n. 11, mar.,1999.

CARLOS, Jairo Gonçalves. Interdisciplinaridade no Ensino Médio: desafios e potencialidades. 2007. 171 f. Dissertação (Mestrado em Ensino de Ciências) - Universidade de Brasília, Brasília, DF, 2007. Disponível em: https://repositorio.unb.br/handle/10482/2961 Acesso em: 25 mar. 2020. 
COSENTINO, Leonardo Antonio Marui. Aspectos Evolutivos da interação homem máquina: tecnologia, computador e evolução humana In: BRASIL. Conselho Regional de Psicologia de São Paulo (Org.). Psicologia \& informática: produções do III. Psicoinfo II. Jornada do NPPI. São Paulo: CRP/SP, 2006. p. 61-71. Disponível em: http://newpsi.bvs-psi.org.br/ebooks2010/en/Acervo files/Psilnfo.pdf. Acesso em: 20 mar. 2020.

CUPANI, Alberto. Filosofia da Tecnologia: um convite. Florianópolis: Editora da UFSC, 2016. Disponível em: https://repositorio.ufsc.br/bitstream/handle/123456789/187613/Filosofia\%20da\%20Tecnologia\%20um\%20convite\%20ebook.pdf?sequence=1. Acesso em: 25 mar. 2020.

DAVIDOFF, Linda. Introdução à Psicologia. São Paulo: Pearson Education, 2001.

DEMO, Pedro. Ambivalências da sociedade da informação. Ciência da Informação, Brasília, v. 29, n. 2, p. 37-42, maio/ago. 2000. Disponível em: http://revista.ibict.br/ciinf/article/view/885/920. Acesso em: 29 mar. 2020.

FORTIM, Ivelise; COSENTINO, Leonardo Antonio Marui. Serviço de Orientação via e-mail: novas considerações. Psicologia Ciência e Profissão, Brasília, DF, v. 27, n. 1. mar. 2007.

Disponível em: http://pepsic.bvsalud.org/scielo.php?script=sci arttext\&pid=S1414-98932007000100014. Acesso em: 20 mar. 2020.

GRANATO, Luísa. Qual é a diferença entre home office e afastamento? Exame. São Paulo, 17 mar. 2020. Disponível em: https://exame.abril.com.br/carreira/qual-e-a-diferenca-entre-home-office-e-afastamento/. Acesso em: 20 mar. 2020.

KUHN, Thomas. A estrutura das revoluções científicas. São Paulo: Perspectiva, 1987.

LANDA DURÁN, Patricia; PEÑALOSA CASTRO, Eduardo. Enseñanza de la Psicología Clínica Conductual, Presencial Vs. En Línea: La Importancia Del Diseño Instruccional. Acta Colombiana de Psicología, v. 12, n. 1, 2009. Disponível em:

http://www.scielo.org.co/scielo.php?script=sci arttext\&pid=S0123-91552009000100010\&lang=pt. Acesso em: 20 mar. 2020.

LEITÃO, Carla Faria; NICOLACI-DA-COSTA, Ana Maria. A Psicologia no novo contexto mundial. Estudos de Psicologia, v. 8, n. 3, p. 421430, 2003. Disponível em: http://www.scielo.br/pdf/epsic/v8n3/19964.pdf. Acesso em: 24 mar. 2020.

MASINI, Elcie Aparecida Fortes Salzano. Enfoque fenomenlógico de pesquisa em educação. In: FAZENDA, Ivani (Org). Metodologia da pesquisa educacional. 9 ed. São Paulo: Cortez, 2004. p. 59-67.

MAROT, Rodrigo Sá Vicente. A Consistência entre as atitudes e as intenções dos internautas em relação à aprovação da terapia Online no Brasil. 2003. Dissertação (Mestrado em Psicologia) - Universidade Gama Filho, Rio de Janeiro, 2003. Disponível em: http://www.psicosite.com.br/pro/tese.htm. Acesso em: 25 mar. 2020.

NEGROPONTE, Nicholas. A vida digital. São Paulo: Companhia das Letras,1995.

PIETA, Maria Adelia. Psicoterapia Online: histórico no Brasil e no mundo. [S.I.:s.n.], 2011. Mesa redonda realizada durante o Seminário Serviços Psicológicos On-Line realizado pelo Conselho Federal de Psicologia. Disponível em:

https://www.youtube.com/watch?v=0a81908Jxfw Acesso em: 25 mar. 2020.

PONTIFÍCIA UNIVERSIDADE CATÓLICA (São Paulo). Laboratório de Estudos de Psicologia e Tecnologias da Informação e Comunicação. [2018]. Disponível em: https://www.pucsp.br/janus/psicoterapia-online. Acesso em: 20 mar. 2020.

RIBEIRO, Wandy. Glossário do coronavírus: entenda os termos que explicam a pandemia. São Paulo, 03 mar. 2020. Disponível em: https://www.ictq.com.br/farmacia-clinica/1368-glossario-do-coronavirus-entenda-os-termos-que-explicam-a-pandemia. Acesso em: 12 abr. 2020.

RODRIGUES, Carmelita Gomes. Aliança terapêutica na psicoterapia breve online. 2014. Dissertação (Programa de Pós-graduação em Psicologia Clínica e Cultura) - Instituto de Psicologia, Universidade de Brasília, Brasília, 2014. Disponível em:

https://repositorio.unb.br/bitstream/10482/16596/1/2014 CarmelitaGomesRodrigues.pdf. Acesso em: 25 mar. 2020

RUFFO, Luciana. Psicoterapia Online: histórico no Brasil e no mundo. [S.I.:s.n.], 2011. Mesa redonda realizada durante o Seminário Serviços Psicológicos On-Line realizado pelo Conselho Federal de Psicologia. Disponível em:

https://www.youtube.com/watch?v=0a81908Jxfw Acesso em: 25 mar. 2020.

SANTANA, Sergio Rodrigues de; MARTINS, Eliane Epifane; SILVA, Laelson Felipe. Mediação social e cultura da informação: acesso e uso das TIC e da informação nos processos de fragmentação da subjetividade. In: ENCONTRO DA ASSOCIAÇÃO DE EDUCAÇÃO E PESQUISA EM CIÊNCIA DA INFORMAÇÃO DA IBERO-AMÉRICA E CARIBE, 9., 2016, Belo Horizonte. Anais [...] Belo Horizonte: UFMG, 2016. Disponível em: https://pt.scribd.com/document/372647015/Anais-Edicic-2016. Acesso em: 15 mar. 2020.

SANTANA, Sergio Rodrigues de. Informação Étnico-Racial no Âmbito dos Programas de Pós Graduação em Psicologia. 2016. Dissertação (Mestrado em Ciência da Informação) - Universidade Federal da Paraíba, João Pessoa, 2016. Disponível em: https://repositorio.ufpb.br/ispui/handle/tede/9701 Acesso em: 15 mar. 2020.

SANTANA, Sergio Rodrigues de; SANTOS, Ozilma Freire dos; FRANCA, Juliana Monteiro; SILVA, Leyde Klebia Rodrigues da. Psicoterapia e orientação on-line: desenvolvendo-se no epicentro do paradigma sociedade da informação. In: SEMANA DE PSICOLOGIA DA UFPB, 4., 2011, João Pessoa. Anais [...] João Pessoa: UFPB, 2011. 1 CD-ROM. 
SISSON, Nathalia; WINOGRAD, Monah. Bachelard e Freud: fenomenotécnica e psicanálise. Arquivos Brasileiros de Psicologia, Rio de Janeiro, v. 64, n. 3, p. 146-162, 2012. Disponível em: http://pepsic.bvsalud.org/scielo.php?script=sci arttext\&pid=S1809-

52672012000300010. Acesso em: 24 mar. 2020.

TARGINO, Maria das Graças; SANTANA, Sergio Rodrigues de; GARCIA, Joana Coeli Ribeiro; SOUZA, Edivânio Duarte de. Do sujeito empático ao sujeito informacional: relações epistemológicas acerca da responsabilidade social na Ciência da Informação. Rev. FSA Periódico do Centro Universitário Santo Agostinho, Teresina, v. 16, n. 3, p. 265-282, maio/jun. 2019.

Disponível em: http://www4.fsanet.com.br/revista/index.php/fsa/article/view/1807. Acesso: 26 mar. 2020.

TAKAHASHI, Tadao (Org.). Sociedade da Informação no Brasil: livro verde. Brasília, DF: Ministério da Ciência e Tecnologia, 2000. Disponível em: https://ivroaberto.ibict.br/bitstream/1/434/1/Livro\%20Verde.pdf Acesso em: 20 dez. 2019.

TEIXEIRA, Olívio Alberto. Interdisciplinaridade: problemas e desafios. Revista Brasileira de Pós-Graduação, n. 1, jul., 2004. Disponível em: http://ojs.rbpg.capes.gov.br/index.php/rbpg/article/view/22/19. Acesso em: 20 dez. 2019.

UNIVERSIDADE FEDERAL DO CARIRI. Coronavírus (Covid-19): Cartilha Educativa. Juazeiro do Norte: UFCA, 2020. Disponível em: https://documentos.ufca.edu.br/wp-folder/wp-content/uploads/2020/03/Cartilha.pdf. Acesso: 27 mar. 2020.

Artigo submetido em: 01/04/2020. Aceito em: 11/04/2020.

\section{UFCA UNIVERSIDADE}

Centro de Ciências Sociais Aplicadas Mestrado Profissional em Biblioteconomia

Este periódico é uma publicação do Programa de Pós-Graduação em Biblioteconomia da Universidade Federal do Cariri em formato digital e periodicidade quadrimestral. 